\title{
Patroklos und Phoinix in der Ilias
}

\author{
HANS-WILHELM NORDHEIDER
}

Meine Damen und Herren, liebe Kolleginnen und Kollegen.

Was ich hier sagen möchte, basiert auf drei Artikeln, die ich für unser Lexikon gemacht habe: Das sind die Artikel Achilleus, Patroklos und Phoinix. Der erste ist eine Jugendsünde, wo ich mehr oder weniger leichtfüßig über die Probleme hinweggehuscht bin; die beiden anderen Artikel - Patroklos und Phoinix kommen, hoffe ich, der Sache näher. In diesen Artikeln finden Sie Stellenangaben, Einzeldiskussionen und Literatur, worauf ich im Folgenden aus Zeitgründen weitgehend verzichte.

Nun zur Sache:

Bekanntlich schildert die Ilias nicht den ganzen 10 Jahre dauernden Kampf um Troia, sondern nur eine Episode von einigen Dutzend Tagen Dauer gegen Ende des Krieges, nämlich den Zorn des von Agamemnon beleidigten Achill -

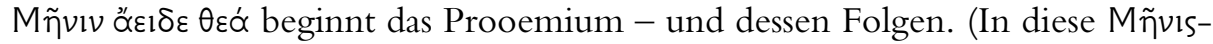
Dichtung wird dann aber der Gesamtkrieg kunstvoll hineingespiegelt, so daß man am Ende doch den Eindruck hat, den ganzen Krieg - und nicht etwa nur eine Achilleis - erlebt zu haben). Dieser Zorn nun wirkt im Hinblick auf das in der Sage vorgegebene Handlungsziel, die Eroberung Troias, als Retardation (und eigentlich ist die ganze Ilias im Rahmen des Gesamtkriegs eine Retardation, die aber natürlich nicht etwa überflüssig ist, sondern das ganze Geschehen in bedeutsamer Verdichtung enthält).

Der beleidigte Achill weigert sich, weiter am Kampf teilzunehmen, spinnt aber nun nicht etwa eine Racheintrige (wie Kriemhild im Nibelungenlied) und konspiriert auch nicht etwa mit den Gegnern (wie das später in historischer Zeit der aus Athen verbannte Alkibiades oder der eine oder andere spartanische König mit den Persern taten), sondern er wartet ab, daß die Griechen ohne seine Mitwirkung scheitern und so die Verkehrtheit ihres Tuns und seinen, Achills, Wert erkennen werden. Seine einzige Gegenaktion besteht darin, seine göttliche Mutter Thetis um Intervention bei Zeus zu bitten, daß der bis auf weiteres den Troern den Sieg verleihe. Er legt die Revanche, die Wieder-

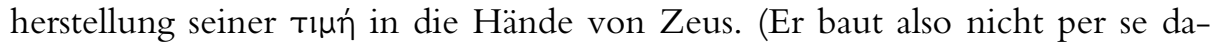
rauf, daß es ohne seine Mitwirkung nicht klappen werde, sondern hilft ein bißchen nach, indem er, salopp ausgedrückt, um den Sieg der Gegner betet). 
Der Dichter steht also vor der Aufgabe, eine Handlung zu bauen, in der der Hauptheld der einen Partei über weite Strecken passiv ist (man könnte dabei an andere Helden des Nichthandelns wie Hamlet oder Wallenstein denken, bei denen aber das Handeln / Nichthandeln als solches problematisiert wird), wo dieser Hauptheld aber andererseits - im Hinblick auf das vorgegebene Handlungsziel - schließlich wieder in den Kampf zurückgeführt werden soll.

Wie geht er dabei vor?

Zum einen, indem er schildert, wie die Griechen ohne Achills Mithilfe nun tatsächlich in sich steigernder Bedrängnis die von diesem angekündigten Mißerfolge erleiden, die in der Erstürmung des Schiffslagers durch die Troer und in Agamemnons defätistischem Vorschlag, den Kriegszug abzubrechen und die Flucht zu ergreifen, gipfeln. Dabei gewinnen - als Ersatz für den abwesenden Achill - andere Helden an Bedeutung: z.B. auf griechischer Seite Diomedes und bei den Troern Hektor.

Zum andern ist aber im Streit zwischen Achill und Agamemnon im Alpha, der Achills Streik verursacht, bereits als Keim der Handlungsstrang der Versöhnung angelegt, und zwar in der Person NESTORS, der als altersweiser, erfahrener Ratgeber sozusagen ein wandelnder Vermittlungsausschuß ist. Er versucht sofort, vermittelnd einzugreifen und die drohende Polarisierung durch eine Kompromißformel, die beiden recht gibt, aber auch beide in ihre Schranken weist, zu überbrücken, dringt aber angesichts des Starrsinns und der zugespitzten Ichbezogenheit, der $\propto \cup ่ \theta \alpha ́ \delta \varepsilon ı$ der Kontrahenten nicht durch.

Nestor bedient sich aber im folgenden nacheinander zweier Akteure, um seinen Vermittlungsversuch fortzusetzen: Das sind PHOINIX und PATROKLOS. Ich gehe jetzt ausdrücklich nicht auf die Frage ein, woher diese Figuren stammen, ob der Dichter, wie nicht unwahrscheinlich, sie bereits in der Tradition vorgefunden hat - dazu finden Sie einiges in den erwähnten Lexikonartikeln. Es geht mir vielmehr um ihre Funktion in der Mñvıs-Handlung der Ilias, zu der sie wesentlich gehören; darum, wie der Dichter sie in die Handlung eingebaut, an die Handlung adaptiert hat und welche Rolle sie dort spielen.

Phoinix tritt zum erstenmal und hauptsächlich nur im Iota auf, ist also wesentlich eine Episodenfigur (auch wenn er außerdem später - im $\Pi, P, T$ und $\Psi$ - noch gelegentlich im Umkreis Achills erwähnt wird). Patroklos dagegen wird - nach unscheinbaren Anfangsnennungen - immer mehr ins Handlungszentrum gerückt und wird zeitweise zum Vertreter Achills und so zu einer im Handeln und Erleiden für den Fortgang der Handlung bestimmenden Zentralfigur.

Auffällig ist nun die Weise, wie beide bei ihrer ERSTNENNUNG unbestimmt und unerklärt aus dem Off auftauchen. Patroklos ist zunächst nur stumme Begleitfigur, wird bei seiner Erstnennung beiläufig nur mit seinem Patronymikon genannt (A 307 verläßt der zornige Achill die Versammlung 


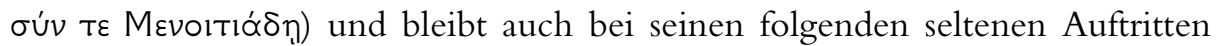
(bei der Übergabe der Briseis sowie bei der Bittgesandtschaft im Iota) stumm im Hintergrund, wobei man mutmaßen kann, daß er dabei die von den Parteien ausgetauschten Argumente hören und auf sich wirken lassen konnte. Und Phoinix wird erstmals - ohne Erklärung des Wer und Woher - von Nestor genannt, als der im Iota in der ởopí vorschlägt, eine Bittgesandtschaft zu Achill zu schicken, um ihn in den Kampf zurückzuführen.

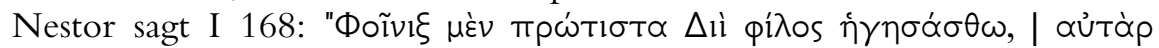

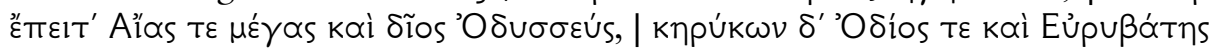
$\alpha^{\prime \prime} \mu^{\prime} \dot{\varepsilon} \pi \varepsilon \dot{\varepsilon} \sigma \omega \nu$." Phoinix ist hier also der erste von fünf Personen in sorgfältig gestaffeltem Nacheinander. - (Das erinnert an die Staffelung von drei Personen

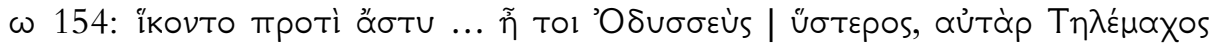

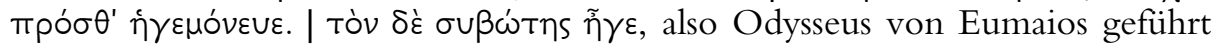
hinterher und Telemach voran).

Die folgende Aktion der Bittgesandten - wie sie zu Achill gehen - wird dann über 30 Verse ausschließlich in Dualen geschildert ('die beiden gingen' usw.), als ob nur zwei Personen statt der zunächst genannten fünf tätig wären. - Diese DUALE sind, wie Sie wissen, eines der meistdikutierten Probleme der Homerphilologie. Die Erklärungsversuche reichen von der Deutung, die schon Aristarch und dann z.B. Von der Mühll u.a. vertraten, daß die Duale sich auf die beiden Hauptgesandten Odysseus und Aias bezögen, wobei

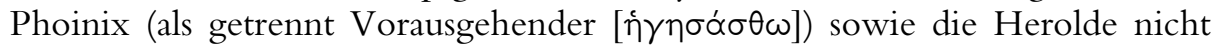
mitgezählt seien, bis zu Wests u.a. Annahme, sie seien die stehengebliebenen Reste einer ursprünglichen Zweiergesandtschaft, in die Phoinix sekundär interpoliert worden sei, und seien mithin auch ein Indiz für die schriftliche Abfassung der Ilias, weil ein mündlicher Dichter solche Unebenheiten wohl harmonisierend ausgeglichen hätte. - Dazu hier nur soviel, daß ich Aristarchs Übersetzung zuneige (Phoinix geht voraus - Odysseus und Aias folgen im Dual); weitere Vorschläge und Diskussion finden Sie samt Literatur im Artikel Phoinix.

Auffällig ist nun, daß Phoinix unvermittelt aus dem Off auftaucht: Weder erfahren wir zunächst, wer er ist, noch, wo er sich zum Zeitpunkt der Versammlung aufhält - jedenfalls ist er nicht bei Achill (zu dessen Entourage er eigentlich gehört, wie wir später erfahren), sondern nimmt offenbar an der Versammlung teil, weil aus dem Folgenden hervorgeht, daß er in Nestors Absicht, Achill zum Mitkämpfen zu überreden, eingeweiht ist.

Erinnern wir uns: Auch Patroklos blieb bei seiner Erstnennung (mit blo-

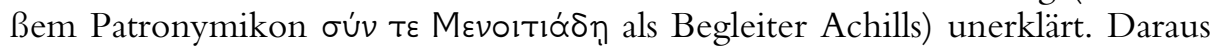
könnte man schließen, daß Patroklos wie Phoinix beide in der der Ilias vorangehenden Sage irgendwie bekannt gewesen sein müssen; darüber lassen sich nur Vermutungen anstellen (dazu Literatur in meinen Artikeln). Und dazu nur die ANMERKUNG, daß wir die Informationen so annehmen müssen - und nur so annehmen können - wie der Dichter sie uns gibt. Und daß ein Epos wie die Ilias - damals wie heute - selten (und vielleicht nur bei philologischen 
Fachtagungen wie der heutigen) auf ein homogenes Publikum von Rezipienten (Hörern / Lesern) mit dem immer gleichen Wissensstand traf und trifft sondern die einen wissen mehr, die anderen weniger von der Vorgeschichte, aber die Dichtung soll auf beide wirken.

Gestatten Sie mir - und entschuldigen Sie bitte - dazu einen kurzen EXKURS, den meine Kollegen sich schon öfter haben anhören müssen: Man könnte das mit der - immer nur stückweisen, nicht ganzheitlichen - Rezeption eines heutigen Kino-Großepos vergleichen - ich meine den Filmmehrteiler 'STAR WARS', der nacheinander in mehreren Teilen - und nicht etwa in der Reihenfolge der Handlungschronologie - veröffentlicht wurde, d.h. in die Kinos kam. (Die Filmemacher begannen vielmehr mit einer Episode aus der Mitte, ließen dann das Happy End mit dem Sieg der Guten folgen und zeigten

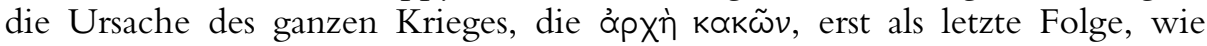
nämlich die Demokratie umkippte zur Tyrannei des, dann durch die ganze Episodenfolge hindurch wirkmächtigen, bösen Imperiums - was sich aber erst am Schluß der Serie aufklärte, zumal es auch keinen den Filmen vorausgehenden Roman gab). Dabei habe ich selber erlebt, daß ich über die Vorgeschichte und Voraussetzungen der Handlung und ihrer Protagonisten viel weniger oder gar nicht - informiert war als meine das Ganze überblickenden Söhne, aber trotzdem das Spiel genossen habe. Das heißt: Die dichterische Schilderung wirkt auch auf Hörer / Leser, die zunächst nicht wissen, wer Patroklos oder Phoinix waren.

Zurück zur Ilias: Wichtiger als Ihr Vorkommen in älterer Sage ist, was der Dichter aus den beiden Figuren macht und wie er sie, auch wenn sie schon in der Tradition vorgegeben gewesen sein sollten, an seine Mñvis-Dichtung adaptiert und wie er uns die Vorgeschichte und Voraussetzungen der beiden Figuren erst dann mitteilt, wenn er sie für ihre Interaktion mit Achill braucht.

Das heißt, die jew. Person wird nicht bei ihrem ersten Auftritt erklärt, indem der Dichter sich informierend an den Hörer / Leser / Rezipienten als Adressaten wendet - wie wir das vom klassischen autonomen / autarken Roman gewohnt sind oder auch vom historischen Sachbuch - sondern die Vorgeschichten von Patroklos und Phoinix werden jeweils als Argumente eingebaut in den Appell, mit dem Phoinix bzw. Patroklos auf Achill einzuwirken versuchen (wobei wir Hörer / Leser sozusagen zu Zeugen eines Bühnendialogs werden - das ist die Technik des Dramas).

Als eines von vielen Gegenbeispielen - nämlich für das Verfahren, eine Person jew. sofort bei ihrem ersten Auftritt dem Leser vorzustellen - nenne ich nur die verdienstvolle und materialreiche Mozart-Biographie von Schenk, der sich die Aufgabe gestellt hat, jede recherchierbare Person zu ermitteln, die Mozart in seinem kurzen Leben getroffen hat. Dabei bringt er es, indem er Mozarts Leben chronologisch nacherzählt, pro Seite auf bis zu sechs in den Handlungsverlauf eingestreute Kurzbiographien, was den Lesetext manchmal zu einem Katalog macht, und erwartet, daß der Leser die jew. einmal vorgestellten Personen dann bei ihren späteren Auftritten im Buch wiedererkennt - 
was schwerfällt und nur mit Hilfe eines Personenregisters gelingt. So geht Homer in der Regel nicht vor. (Höchstens erhält der eine oder andere 'Kleine Kämpfer' bei seinem einmaligen Auftritt angesichts seines Todes mal solch eine Kurzbiographie).

Wer Phoinix ist und welche Vorgeschichte er hat, erfahren wir erst, wenn er - in seiner Rede an Achill - mit Hilfe eben dieser Vorgeschichte und der Hervorhebung bestimmter biographischer Details auf Achill einzuwirken versucht, wieder am Kampf teilzunehmen. Diese Rede steht zwischen den Reden des Odysseus und Aias: ist der Mittelteil und auch der Höhepunkt des 'Redetriptychons' der Bittgesandtschaft (wie Wilamowitz das ausdrückt) und ist besonders reich an erzählerischen Elementen: Zunächst zwei Geschichten von fruchtlosem / kontraproduktivem Zorn - die eine aus Phoinix' eigener biographischer Vorgeschichte, wie er, von seinem Vater zur Kinderlosigkeit verflucht, daran gehindert werden mußte, diesen umzubringen (ebenso mußte Achill von Athene zurückgehalten werden, im Affekt Agamemnon umzubringen); - dann die Geschichte vom fruchtlosen Zorn des Meleager als epische Einlage. Beide Zornesgeschichten sind - vom Dichter an die Situation adaptierte - Exempla, die Achill die Fruchtlosigkeit seines eigenen Zornes vor Augen führen und ihn zur Umkehr bewegen sollen. Schließlich die Allegorie von den Nı̊á, den 'Bitten' - als personifizierten Zeustöchtern - denen Achill sich nicht verweigern dürfe. Darein verwoben als Erinnerung, die die Nähe und Vertrautheit des Sprechers evozieren soll, wie Phoinix als íḱtᄁns an den Hof von Achills Vater Peleus kam, Achills Erzieher wurde und diesen als Kleinkind auf seinem $\mathrm{Schoß}$ fütterte.

Lassen Sie mich en passant nur auf ein Detail in Phoinix' Biographie hinweisen: Wie er, von seiner eifersüchtigen Mutter überredet, mit der $\pi \propto \lambda \lambda \propto$ кís, der Konkubine seines Vaters, schläft, um diese dem Alten abspenstig zu machen, und daraufhin von dem erzürnten Vater zur Kinderlosigkeit verflucht, also gleichsam kastriert wird. Das klingt wie eine Herodot-Novelle mit nahöstlicher Färbung und hat direkte Parallelen im Alten Testament (worauf schon Brown, Israel und Hellas und St. West hingewiesen haben): Da 'geht' (2 Samuel) der rebellische Absalom - in einem auf dem Dach des väterlichen Palastes erbauten Zelt - 'vor allem Volk' zu den Frauen seines von ihm vertriebenen Vaters David 'ein', damit Israel erkenne, daß er der neue König ist; er nimmt also zum Zeichen des Machtwechsels den väterlichen Harem in Besitz. - Hinter Phoinix' Beischlaf mit der väterlichen Konkubine scheint also, ins privat Monogame einer persönlichen Intrige umgebogen, ein nahöstlicher Königssturz zu stehen. - Andere Versionen, wo Phoinix fälschlich von der Frau des Vaters des Beischlafs beschuldigt wird - also das alttestamentarische Potiphar-Motiv (wie bei Hippolytos und Phaidra) - findet man bei Apollodor und fand man wohl auch im verlorenen Phoinix des Euripides. (Dazu weiteres im Artikel Phoinix). - Jedenfalls scheint solch ein nahöstlicher Zug zu 'Homer als Dichter der orientalisierenden Epoche' (wie Walter Burkert formuliert) zu passen. Und vergessen wir dabei nicht, daß Фoĩvı - trotz mancher von Müh- 
lestein probierter ingeniöser Assoziationen - zunächst 'der Phöniker' heißt, der - vom Vater gleichsam kastriert - den kleinen Achill auf seinem Schoß füttert

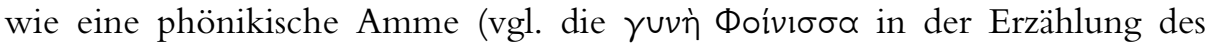
Eumaios; und vgl. andererseits als weiteren nahöstlichen Personennamen noch den Greis namens Aiyúntıos, der in der Volksversammlung auf Ithaka auftritt).

Phoinix' Rede bleibt nicht ohne Wirkung auf Achill: Er schwächt die zuvor geäußerte Absicht, heimzufahren, jetzt ab zur möglichen Alternative (I 619

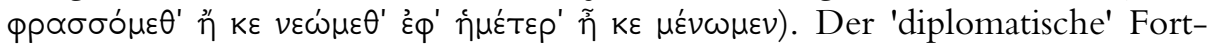
schritt der Verhandlungen mit Achill ist also wesentlich dem Appell von Achills väterlichem Freund und Vertrauten Phoinix zu verdanken. Insofern ist die von Nestor initiierte Bittgesandtschaft - die eigentlich eine Kombination aus zwei formal und grammatisch unterschiedenen Aktionen war: nämlich der (durch den Dual ausgedrückten) Aktion der beiden 'offiziellen Botschafter' Odysseus und Aias einerseits und des Appells des vertrauten Freundes andererseits - nicht ganz fruchtlos geblieben. Im übrigen bleibt Phoinix, während die beiden anderen zu den Griechen zurückkehren, jetzt bei Achill - ist gleichsam zu diesem zurückgeführt - wohin er gehört.

War Phoinix von Nestor - und vom Dichter - unvermittelt und unerklärt als Vermittler ins Spiel gebracht worden, so wird das Eingreifen des PATROKLOS gründlicher motiviert. Beidemale spielt der Dichter ein ähnliches The$\mathrm{ma}$ - das des von Nestor beauftragten Vermittlers zwischen Achill und den Griechen - durch, aber das zweitemal, bei Patroklos, hat er mit seinem Vermittler mehr vor und bemüht sich, dessen Eintritt aus der Latenzphase in die aktive Phase sorgfailtiger zu motivieren.

(Man könnte an Goethes Bemerkung anläßlich der Theaterauffuihrungen von Schillerschen Dramen denken, Schiller in seinem ungeduldigen geistigen Höhenflug sei manchmal etwas großzügig oder nachlässig im Motivieren von Bühnenaktionen gewesen, so daß er manchmal im Interesse des kritischen Publikums habe nachmotivieren müssen). - Hierzu noch ein iliadisches Beispiel für eine solche HARMONISIERENDE NACHMOTIVIERUNG: $\Lambda$ 771, als - in Nestors Erzählung - Nestor und Odysseus zur Anwerbung der Kriegsteilnehmer zu Peleus nach Phthia kommen, läßt der Dichter sie dort auch Menoitios antreffen - nicht, weil der mit seinem Sohn dauernd vom heimischen Opous zu Peleus nach Phthia emigriert wäre (wie Leaf im Kommentar zu meinen scheint), sondern weil der Dichter ihn dort braucht, damit Nestor im folgenden die Mahnungen beider Väter an ihre Söhne zitieren und damit jetzt ad hoc Patroklos beeinflussen kann. (Ebenso ist auch die Mutter Thetis mal von Peleus getrennt, mal sind sie und der Vater als gemeinsam auf den Sohn Achill wartendes Elternpaar gedacht). - Eine ähnliche, wenn auch oberflächliche, Motivierung - richtiger Behauptung - von Phoinix' Anwesenheit bei der Heeresversammlung - also etwa die Einfügung: 'Phoinix, der gerade hier ist, soll vorausgehen' - würde uns beruhigen und uns viele philologische Erörterungen ersparen. 
Entsprechend der bedeutsameren Rolle, die Patroklos in der Ilias spielt, hatte bereits $\Theta 475$ Zeus prophetisch Patroklos' Tod mit Achills Kampfeintritt und Hektors Ende zusammengesehen; eine zweite Prophezeiung folgt O 63ff. - Patroklos' zukünftiger Tod wird also - wie der nachiliadische Tod Achills $\mathrm{zu}$ einem der strukturierenden Fernbezüge, die das Epos durchorganisieren und verklammern. Es ist aber tragische Ironie, daß Patroklos' Tod zwar uns, dem Publikum, mitgeteilt wird, aber - anders als der des Achill - weder ihm selber noch Achill bewußt ist.

Patroklos' Eintritt in die aktive Phase wird vom Dichter prophetisch mit einer Unheilsüberschrift angekündigt. Angesichts der von den Troern verfolgten zurückflutenden Griechen schickt Achill Patroklos aus, sich nach einem Verwundeten zu erkundigen; der - Patroklos - ( 1604$)$ : है

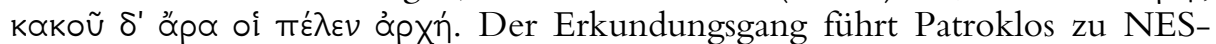
TOR - wieder ist Nestor der Iniator der Gegenaktion - wo aber die Erkundigung nach dem Verwundeten bald zu der Nebensache wird, die sie von Anfang an war. Vielmehr versucht Nestor erneut - diesmal durch eine längere Rede an Patroklos, in der er auch Ermahnungen der Väter Menoitios und Peleus zitiert, also die Situation des Kriegsanfangs evoziert - Achill in den Kampf zurückzuführen, und gibt dann als Alternative Patroklos den entscheidenden Plan ein, in den Waffen Achills und an dessen Stelle in den Kampf einzugreifen - wobei die Troer durch den Anschein, Achill kämpfe wieder mit, getäuscht und in Schrecken versetzt werden sollen. Wie wir wissen, funktioniert dieser Plan radikaler, als es sich die Initiatoren vorstellen konnten. Achill läßt Patroklos an seiner statt in den Kampf ziehen; der bekommt seine Aristie, wie sie nur erstrangigen Helden zukommt, überschreitet dabei aber auch seine ihm von Achill gesetzte Grenze, rechtzeitig umzukehren, und fällt in Parallele und Vorwegnahme von Achills eigenem Tod - durch Apoll, Hektor und Euphorbos - dessen Beteiligung die direkte Rache durch Menelaos ermöglicht - wobei Euphorbos' Hirtenname und seine charitinnengleichen, reichgeschmückten Haare (P 51) an Paris, den Achilltöter der Aithiopis, erinnern.

Durch Patroklos' Tod verwandelt sich der Zorn der beleidigten Ehre bei Achill in Rachezorn gegen Hektor und treibt ihn wieder in den Kampf.

Patroklos hat nach seinem Tod noch einen letzten Auftritt: $\Psi$ 65ff. erscheint Patroklos' Totengeist (seine $\left.\psi \cup \times \eta^{\prime}\right)$ dem Achill, kündigt diesem dessen eigenen bevorstehenden Tod an und bittet um gemeinsame Bestattung - eine Szene, die an die Erscheinung des toten Enkiddu im Gilgamesch-Epos denken läßt. Zur Motivierung dieses Wunsches erinnert er Achill an ihre gemeinsame Verbundenheit - erst hier erfahren wir den Anfang ihrer beider Vertrautheit, wie er wegen eines Totschlags als íḱ́tns an den Hof des Peleus kam und von diesem zu Achills $\theta \varepsilon p o ́ n t \omega v$ gemacht wurde.

FASSEN WIR ZUSAMMEN: Phoinix und Patroklos sind Akteure innerhalb des Mñvıs-Themas der Ilias. Sie sind in ihrer Funktion Parallelfiguren, insofern beide im Auftrag Nestors den erzürnten Achill wieder in den Kampf 
zurückführen sollen. Zu diesem Zweck appellieren sie nicht spontan an Achill, wie es ihnen bei ihrer Vertrautheit und Zugehörigkeit zum Haushalt Achills möglich wäre (I 190 sitzt Patroklos schweigend bei Achill und hört seinem Gesang zu); der Dichter läßt sie vielmehr jeweils den Umweg über Nestor den iliadischen Prototyp des Vermittlers - nehmen, mit dessen Instruktionen ausgerüstet sie dann Achill zu beeinflussen suchen, wobei sie Nestors Argumente zusätzlich durch die Schilderung menschlicher Vertrautheit und Nähe bereichern und emotional verlebendigen. Erst im Fortgang dieser Handlung werden ihre Vorgeschichten, Rollen und Charaktere sukzessiv entfaltet, d.h. auf verschiedene Teile der Handlung verteilt und in den Reden der Beteiligten enthüllt, wo sie jeweils argumentativ und emotional auf Achill einwirken sollen.

Beider Aktionen sind sozusagen - musikalisch - die zweimalige Durchführung eines ähnlichen Themas. Beide sind ältere Vertraute und Erzieher Achills - und ersetzen insofern bei Achill, der ja nach Thetis' Weggehen mutterlos ist, den vor- und außeriliadischen Kentauren Cheiron als Erzieher. Dabei bleibt Phoinix in der Ilias episodisch, Patroklos dagegen wächst über die Rolle des bloßen Vermittlers, wie es Phoinix ist, hinaus; er wird handelnd und erleidend zum Vertreter Achills und nimmt auch dessen nachiliadischen Tod vorwegwie er auch den nachiliadischen Tod des anderen Achillfreundes Antilochos vorwegnimmt (worüber W. Kullmann Wichtiges gezeigt hat). Mithin verwendet der Dichter auf seine Integration in die Handlung mehr Sorgfalt, kündigt auch seinen Tod durch leitmotivische Prophezeiungen an.

Die Schilderung der menschlichen Beziehungen, vorwiegend durch die Reden, die Phoinix und Patroklos an Achill richten und wie sie nur von vertrauten Freunden kommen können, gehört - neben solchen Höhepunkten wie Hektors Abschied von Andromache oder Achills und Priamos' Begegnung im $\Omega$ - zum seelisch Reichsten, was die Ilias zu bieten hat.

\section{Als Anhang \\ Zur Genese der „Bremer Stadtmusikanten“ \\ Ein analytisch-neoanalytisch-strukturalistisch-allegoretischer Beitrag zur Homerischen Frage}

Hans-Wilhelm Nordheider

Vier unter dem gebräuchlichen, wenn auch nicht wissenschaftlich exakten Begriff „Haustiere“ subsumierbare Wirbeltiere, davon drei zu den Säugern (Mammalia), eines zu den Vögeln (Aves) gehörig, gelangen, jeweils durch ein

* Der folgende Beitrag desselben Autors, schon 1983 im Privatdruck einer Festschrift für Eva-Maria Voigt erschienen, steht hier noch einmal, um zu demonstrieren, daß Lexikonarbeit auch Spaß machen kann. 
ähnliches Schicksal aus ihren Behausungen vertrieben, auf dem Weg nach Bremen, wo sie Stadtmusikanten werden wollen, in ein Räuberhaus inmitten eines dunklen Waldes, vertreiben die dort Befindlichen und bleiben dort.

Diese scheinbar so einfache und schlüssige, weil seit den Brüdern Grimm vertraute Geschichte verbirgt unter ihrer Oberfläche kindlicher Harmlosigkeit mancherlei Probleme.

\section{Die Personen der Handlung}

1. Wie verträgt sich zunächst die Eigenart der vier Tiergestalten mit ihrem Plan, Stadtmusikanten zu werden? Schon auf den ersten Blick erkennt man, daß Esel und Katze typologisch hervorragend in die Kategorie der musikantischen Tiere passen (erinnert sei nur an das bekannte Volkslied vom Sängerwettstreit zwischen Kuckuck und Esel oder an den mit leidvoller Erfahrung gestörter Nachtruhe verbundenen Begriff der Katzenmusik); dagegen kommen Hund und Hahn, wenn auch wie viele andere Tiere mit charakteristischen Stimmen begabt, wohl eher aus einem anderen Zusammenhang (s.u. I 2, II 3).

2. Esel und Katze, ersterer schon in Apuleius' Metamorphosen und in mittelalterlichen Schwänken fahrender Scholaren mit häufigem Schauplatzwechsel auftretend, letztere bekannt für charakterliche Unabhängigkeit und Distanziertheit, gehören zum Typ der vagantischen Tiere; wohingegen der treue Hund und der wachsame Hahn geradezu Symbole haus- und hofverbundener Beständigkeit und Immobilität darstellen.

3. Der Repräsentant der sprichwörtlichen Eselsdummheit und die listig geschmeidige Katze bilden ein konträres, in gegenstrebiger Fügung verbundenes Paar nach Art von Wolf und Fuchs, Kain und Abel, David und Goliath, Faust und Mephisto, Adam und Eva, Don Quijote und Sancho Pansa oder Sherlock Holmes und Doktor Watson, das man sich gut als Helden einer abenteuerlichen Vagantengeschichte nach Art pikaresker Romane vorstellen kann; wobei die beiden anderen, Hund und Hahn, nur schwer integrierbar wären.

4. Bisher ergibt sich also: Esel und Katze eignen sich typologisch gesehen sowohl als Freundespaar wie für das unserer Geschichte zugrundeliegende Musikanten- und Vagantenmotiv bestens, während Hund und Hahn in diesem Zusammenhang anscheinend sekundär sind.

\section{Die Handlungsstränge}

1. Jedem unvoreingenommenen Leser muß der Widerspruch auffallen, der zwischen dem die erste Hälfte der Erzählung beherrschenden (auch titelgebenden) Plan, nach Bremen zu gehen (B) und dort Stadtmusikant zu werden (M) - im folgenden ,Ziel': $Z^{\mathrm{B}+\mathrm{M}}$ genannt - und dem Ende der Geschichte besteht, wo dieser Plan von den Tieren völlig vergessen zu sein, oder besser: überhaupt 
nicht existiert zu haben scheint. Stattdessen ist nun ein ,hellerleuchtetes Räuberhaus" $(\mathrm{H})$, in das die Tiere unter Vertreibung der dort schmausenden Räuber eindringen, Mittelpunkt und Ziel der Handlung $\left(\mathrm{Z}^{\mathrm{H}}\right)$.

2. Die Vertreibung der Räuber aus dem Haus nun, in der traditionellen Lesart als Vertreibung aus dem Räuberhaus, oder marxistisch als Expropriation der Exproprieteure dargestellt, ist ihrerseits als Motiv nicht unproblematisch: Wieder sei an das gesunde Volksempfinden des unvoreingenommenen Lesers appelliert, der sich unter einer Vertreibung von Räubern zunächst einmal die Vertreibung aus dem geraubten, d. h. gewaltsam und widerrechtlich besetzten Eigentum anderer, vorstellt. An unsere Erzählung ist also die Frage zu richten: Wie sind die Räuber in das sog. Räuberhaus gekommen, in dem sie als festlich Tafelnde (,Schmausende' und nicht ,Hausende') geschildert werden.

3. Hier sei nun an den oben (I 2) mitgeteilten Gedanken angeknüpft, daß Hund und Hahn ihrem Typus nach keine vagantischen Musikanten, sondern Wächter sind, die man sich, wie durch unzählige Literaturbeispiele zu belegen, am besten als Hüter und Bewohner von Haus und Gehöft vorstellen kann. Es scheint nun sehr plausibel, diese Hüterfunktion in irgendeiner Weise auf das sog. Räuberhaus, d. h. das von den Räubern besetzte Haus, zu beziehen. Dann läßt sich folgende ursprüngliche Version erschließen: Hund und Hahn nehmen an der Rückeroberung ihres von Räubern besetzten Hauses teil.

\section{Die Genese}

Fassen wir zusammen:

1. Wir haben es mit zwei verschiedenartigen Tierpaaren zu tun, dem musikantisch-vagantischen Duo Esel-Katze und dem wächterisch-bodenständigen Paar Hund-Hahn.

Beide Paare sind in ihrer Gegensätzlichkeit zunächst kaum in einer gemeinsamen Handlung vorstellbar, fügen sich aber jeweils sehr gut in die beiden Hälften unserer Geschichte: Esel und Katze in die Wanderung nach Bremen samt Musikantenprojekt, Hund und Hahn in die Rückeroberung der Hausherrnschaft.

2. Die Diskrepanzen lassen sich am besten erklären, wenn man folgende Genese für unsere Geschichte annimmt:

a) Am Anfang steht die abenteuerliche, pikareske Vagantengeschichte vom gegensätzlichen Freundespaar Esel und Katze $\left(\mathrm{V}^{\mathrm{Es}+\mathrm{Ka}}\right)$, die nach Bremen wandern, um dort Stadtmusikanten zu werden, und - so darf man vermuten - auf dieser Wanderung allerlei (leider nicht überlieferte) Abenteuer (Episoden: $\mathrm{E}^{\mathrm{XY}}$ ) erleben.

b) Daneben steht, zunächst selbständig, die Hausgeschichte von Hund und Hahn $\left(\mathrm{H}^{\mathrm{Hu}+\mathrm{Ha}}\right)$, die ihrem (ebenfalls der Überlieferung zum Opfer gefallenen) Herrn (Dom.) helfen, die eingedrungenen Räuber zu vertreiben und die Hausherrnschaft zurückzuerobern. - Die Parallele zur Odyssee ist hier unüber- 
sehbar, der Rückschluß auf eine evtl. ursprüngliche Teilnahme des Hundes Argos an Odysseus' Kampf gegen die Freier erlaubt. - Diese Rückeroberung erscheint in unserer Geschichte aufgespalten und umgeformt in zwei Aktionen: einerseits als Vertreibung der schmausenden Räuber, andererseits als Zurückschlagung des Räuberspähers am Schluß (ein an die Dolonie der Ilias erinnerndes Nocturno), wobei Einzelheiten noch die offenbar kakophone Drastik der Vorlage (z.B. die in , abenteuerlichem Stil' geschilderten Verwundungen des Spähers durch die Tiere oder deren Stimmengeheul) aufblitzen lassen.

c) Aus diesen beiden selbständigen Geschichten V und $\mathrm{H}$ schuf ein Erzähler die seit den Brüdern Grimm geläufige Fassung der „Bremer Stadtmusikanten" (BM), indem er, vielleicht unter dem Einfluß bildlich dargestellter Tierpyramiden (erinnert sei an nahöstliche Kompositpfeiler aus Tierfiguren, südindische Tempeltürme und indianische Totempfähle), das ursprüngliche Vagantenpaar Es + Ka durch Hinzufügung der ursprünglichen Wächter $\mathrm{Hu}+$ $\mathrm{Ha}$ zum Quartett Es $+\mathrm{Ka}+\mathrm{Hu}+\mathrm{Ha}$ erweiterte (also die seither berühmte und als Denkmal am Bremer Rathaus aufgestellte Tierpyramide schuf) und andererseits die Hausgeschichte $\mathrm{H}$ (unter Verkürzung auf die Räubervertreibung $\mathrm{R}$ ) in die Vagantengeschichte $\mathrm{V}$ einschaltete, wobei diese (R) andere Abenteuerepisoden $\left(\mathrm{E}^{\mathrm{XY}}\right)$ und auch das Handlungsziel $\left(\mathrm{Z}^{\mathrm{B}+\mathrm{M}}\right)$ verdrängte und so aus einer, ursprünglich vielleicht beabsichtigten, Episode $\left(\mathrm{E}^{\mathrm{H}}\right)$ selber zum Handlungsziel $\left(\mathrm{Z}^{\mathrm{H}}\right)$ wurde. Es ergibt sich also:

$$
\mathrm{V}^{\mathrm{Es}+\mathrm{Ka}}+\mathrm{H}^{\mathrm{Hu}+\mathrm{Ha}}=\mathrm{BM}^{\mathrm{Es}+\mathrm{Ka}+\mathrm{Hu}+\mathrm{Ha}}
$$

oder genauer:

$$
\mathrm{V}^{\mathrm{Es}+\mathrm{Ka}}-\left(\mathrm{E}^{\mathrm{XY}}+\mathrm{Z}^{\mathrm{B}+\mathrm{M}}\right)+\mathrm{R}\left(=\mathrm{H}^{\mathrm{Hu}+\mathrm{Ha}}-\mathrm{X}^{\text {Dom.etc. }}\right)=\mathrm{BM}^{\mathrm{Es}+\mathrm{Ka}+\mathrm{Hu}+\mathrm{Ha}}
$$

wobei $\mathrm{X}$ und $\mathrm{Y}$ verlorengegangene Teile (Episoden: E, Figur des Hausherrn: Dom. usw.) bezeichnen sollen.

\section{Der Dichter}

Abschließend noch ein Wort über die Weise dieser Verknüpfung:

Die Brücke oder Kompositionsfuge zwischen der Vagentengeschichte (V) und der Rückeroberung (R) bildet der berühmte, an kafkaeske Frustrationsobsessionen erinnernde Satz: „Sie konnten aber die Stadt Bremen an diesem Tage nicht erreichen und übernachteten deshalb in einem dunklen Wald“. Hier ist einerseits das im folgenden verdrängte Handlungsziel Bremen $\left(Z^{\mathrm{B}+\mathrm{M}}\right)$ ein letztes Mal genannt, andererseits die ab jetzt dominierende Räubervertreibung $\left(Z^{\mathrm{H}}\right)$ eingeleitet; die Passage muß also dem vereinigenden Dichter der Endfassung gehören.

An dieser kompositionell höchst empfindlichen Stelle nun steht der Hinweis auf den ,dunklen Wald', der blitzartig klarmacht, von welcher Absicht sich der Erzähler von BM leiten ließ: Unüberhörbar ist hier das Zitat des Eingangs von Dantes Divina Commedia: 
Nel mezzo del cammin di nostra vita mi ritrovai per una selva oscura

wodurch sich der 'dunkle Wald', in dem sich unsere vier Vaganten, die Vertreter kraftvoller Dummheit (Es), listiger Geschmeidigkeit (Ka), Treue (Hu) und Wachsamkeit $(\mathrm{Ha})$, verirrt haben, als der den Menschen umstrickende Bereich von Irrtümern und Leidenschaften erweist.

In diesem Wald nun sieht der Hahn, Vogel der Wachsamkeit, in der Ferne das Licht des Räuberhauses brennen, das für die Vier zum Ort der Bewährung und schließlich nach der Inbesitznahme zum himmlischen Jerusalem wird, neben dem das irdische Ziel einer Beamtung in Bremen keinen Bestand hat.

Es war also kein bloßer kompilierender Redaktor, sondern ein komponierender Dichter und tiefer Denker, in dessen Weltbild der dunkle Wald der Verstrickung wie das helle Licht der Gnade ihren Platz haben, und zudem, wie die monumentale Idee der Tierpyramide zeigt, ein Visonär von grandioser Gestaltungskraft, der aus den handfesten Abenteuer- und Kampfgeschichten V und H die „Bremer Stadtmusikanten“ schuf und damit das zeitlose Thema der peregrinatio, des Weges der Seele durch Verstrickung und Bewährung zur leuchtenden Himmelsstadt, neu und eindrucksvoll gestaltete.

Nachtrag: Wieweit bei unserer Geschichte noch das Thema der retardierten Heimkehr, also eine Nostendichtung, als Vorlage eine Rolle spielte, als deren Helden man sich am liebsten eine Brieftaube vorstellen würde, muß weiterer Forschung vorbehalten bleiben. 